\title{
Phospholipid/cholesterol/decanethiol mixtures for direct assembly of immunosensing interfaces
}

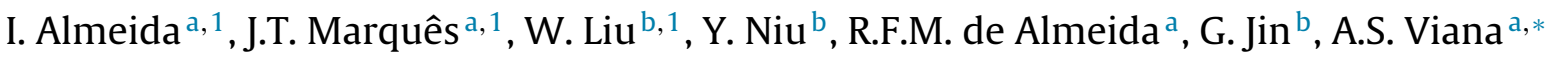 \\ a Centro de Química e Bioquímica. Faculdade de Ciências, Universidade de Lisboa, Edifício C8, Campo Grande, 1749-016 Lisboa, Portugal \\ ${ }^{\mathrm{b}}$ Institute of Mechanics, Chinese Academy of Sciences, Beijing 100190, China
}

\section{A R T I C L E I N F O}

\section{Article history:}

Received 31 July 2015

Received in revised form 13 October 2015

Accepted 29 October 2015

Available online 3 November 2015

\section{Keywords:}

Planar hybrid supported lipid bilayers

Thiol-gold linkages

Antibody covalent coupling

Inhibition of nonspecific adsorption

Immunosensors

Surface plasmon resonance

\begin{abstract}
A B S T R A C T
In this work, a simple yet robust method to prepare lipid-based biosensing interfaces on gold using common lipids (a phospholipid and cholesterol) and an alkanethiol is reported. The lipids were carefully chosen to tailor the biophysical properties of the bilayer. The simplicity of the method relies on the incorporation of a small percentage of decanethiol in the lipid vesicles for a direct formation of a thiol-linked supported lipid bilayer, which is advantageous in several respects. It prevents the use of specially synthesized thiolipids and preserves the natural fluidity and dynamics of the lipids. As a consequence the whole arrangement is extremely stable regarding ionic strength changes and solution flow during surface plasmon resonance experiments. Moreover, we show that this interface is very effective on suppressing the nonspecific adsorption of proteins on the surface, and enables the covalent attachment of the recognition antibody. The subsequent detection of specific interaction toward antigen was monitored in real-time by SPR and confirmed by ellipsometric measurements. This lipid-based biosensing platform is versatile and can be adapted to the biorecognition reaction of interest.
\end{abstract}

(c) 2015 Elsevier B.V. All rights reserved.

\section{Introduction}

The development of new and enhanced biosensing devices is challenging, and the use of lipid-based platforms is a highly promising approach since they provide a biomembane-like environment. It is biocompatible, prevents denaturation of the transducing protein through contact with the metal surface, and may inhibit nonspecific binding [1-8]. The formation of planar lipid-based biomimetic platforms on gold surfaces has aroused the interest of many researchers [9-12]. One of the most limiting features of this type of assemblies has been the stability of the supported lipid bilayer (SLB) on gold which has led to complex approaches that require especially synthesized molecules, namely polyethylene glycol (PEG)-derivatives [13,14], thiolipids [6,15], biotinylated lipids [14-17] or other tether layers $[18,19]$. In this work we describe an alternative approach that takes full advantage of both the biophysical and the chemical properties of lipids in order to build a simple and efficient immunosensing platform. A binary mixture of 1,2-dipalmitoyl-sn-glycero-3-phosphocholine (DPPC)/cholesterol (Chol) (70:30) was employed. The phase diagram for this mixture shows that at the lipid molar proportions

\footnotetext{
* Corresponding author.

E-mail address: anaviana@fc.ul.pt (A.S. Viana).

1 These authors contributed equally to this work.
}

used the system is in the liquid ordered phase [20-23], which presents high mechanical resistance [24] and molecular packing but at the same time is fluid. This fluidity will not only allow for the proper functional dynamics of the biosensing proteins but also facilitate the formation of a planar and continuous lipid bilayer from lipid vesicles. The liquid ordered phase is the most representative of plasma membrane in human cells and is very stable regarding temperature and pressure changes [25]. The lipid vesicles contain also $5 \%$ of decanethiol in order to promote a strong attachment of the lipid bilayer to the gold surface (Fig. 1). Once the SLB is formed, the direct activation of the phosphate groups, present in the lipids' polar head group, enables their covalent coupling to human immunoglobulin $\mathrm{G}$ ( $\operatorname{IgG}$ ) (Fig. 1) through amine groups, using $\mathrm{N}$-(3dimethylaminopropyl)- $N^{\prime}$-ethylcarbodiimide hydrochloride (EDC) and $\mathrm{N}$-hydroxysuccinimide (NHS) coupling agents as previously used to activate phosphate groups in double-stranded DNA [26]. The performance of the interface was assessed using the wellestablished anti-IgG / IgG biorecognition.

\section{Experimental}

\subsection{Chemicals}

DPPC was purchased from Avanti Polar Lipids (Alabaster, AL). Chol, minimum 99\%, 11-mercaptoundecanoic acid (MUA), min- 


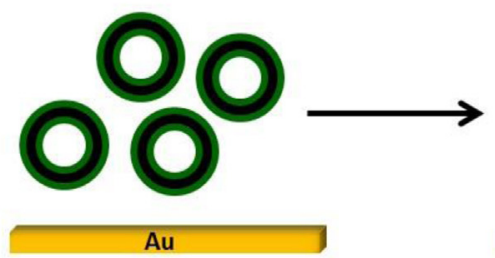

$\mathrm{Au}$
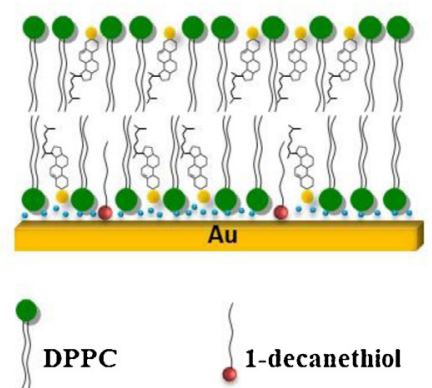
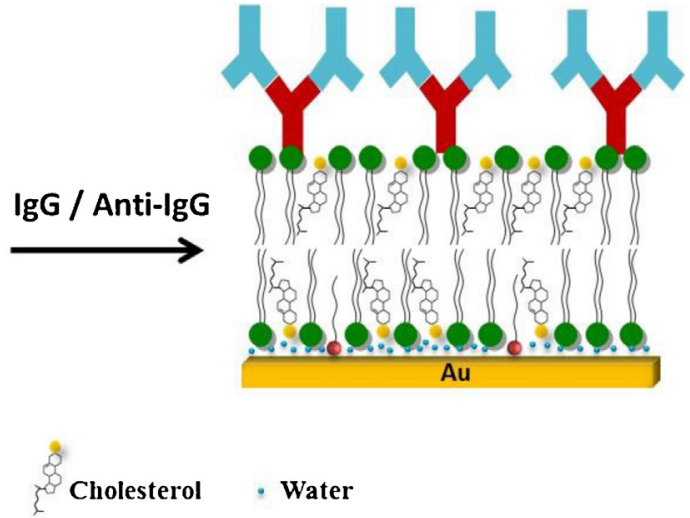

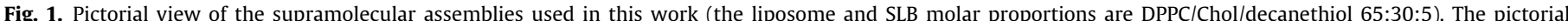

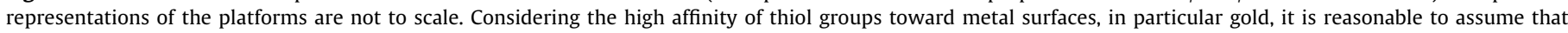

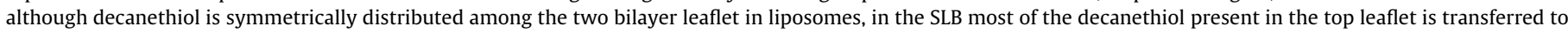
the bottom leaflet. For simplicity all the antibodies were represented with the same relative orientation.

imum 95\%, 1-decanethiol, minimum 96\%, NHS, minimum 98\%, EDC, minimum $98 \%$, and all other reagents, analytical grade, were acquired from Sigma-Aldrich. The $10 \mathrm{mM}$ Hepes, $150 \mathrm{mM} \mathrm{NaCl}$ buffer was prepared with ultrapure Milli-Q water (18.2 M $\Omega . \mathrm{cm}$ ) and used for hydration of lipid and deposition of vesicles. Phosphate

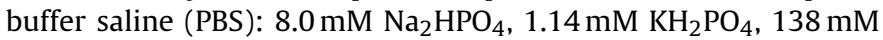
$\mathrm{NaCl}$, and $2.7 \mathrm{mM} \mathrm{KCl}, \mathrm{pH} 7.4$, with or without $0.05 \%(\mathrm{v} / \mathrm{v})$ of Tween 20 , prepared with ultrapure Milli-Q water was used for the preparation of protein solutions and as a washing solution directly in SPR experiments.

Antibodies for immunosensing, polyclonal human IgG and polyclonal anti-human $\operatorname{IgG}(\mathrm{H}+\mathrm{L})$ secondary antibody from goat, as other proteins, such as bovine serum albumin (BSA), and casein blocking buffer (BB) were obtained from Sigma-Aldrich and used without further purification.

\subsection{Preparation of gold surfaces}

In this work, two types of gold have been used, since for SPR measurements only polycrystalline gold substrates (with a granular morphology) are available, whereas for atomic force microscopy (AFM) atomically flat $\mathrm{Au}(111)$ should be employed in order to clearly depict small details in the topography of the films under study.

For SPR experiments, thin layer gold $(50 \mathrm{~nm})$ coated glass slides were purchased from Analytical $\mu$-Systems. Prior to use the slides were rinsed with water and ethanol and cleaned in a UV chamber for $45 \mathrm{~min}$. For ellipsometry and AFM analysis, gold substrates manufactured by Arrandee consisting of a thin film $(200 \mathrm{~nm})$ of gold on borosilicate glass $\left(1.1 \times 1.1 \mathrm{~cm}^{2}\right)$ with a chromium undercoat $(2-4 \mathrm{~nm})$ were used and cleaned in piranha solution $\left(\mathrm{H}_{2} \mathrm{SO}_{4} / \mathrm{H}_{2} \mathrm{O}_{2}=3: 1, \mathrm{v} / \mathrm{v}\right)$ and in a UV chamber for $45 \mathrm{~min}$ as described elsewhere [5,27]. These latter substrates were also flameannealed after which they acquired a preferential (111) orientation, as proved by scanning tunneling microscopy measurements [28].

\subsection{Gold modification with MUA and decanethiol self-assembled monolayers (SAMs)}

SAMs composed of MUA or decanethiol, were tested against the nonspecific binding of proteins. MUA SAM was also used to prepare a SLB, since the terminal $\mathrm{COOH}$ groups provide a hydrophilic interface suitable for phospholipid deposition. In this work, decanethiol was also employed as a constituent of liposomes for the ready preparation of SLB on bare gold.
SAMs on gold were formed through immersion of the gold slides in a $1 \mathrm{mM}$ solution of each thiol (MUA or decanethiol) in ethanol for $18 \mathrm{~h}$, followed by abundant rinsing of the modified substrates with ethanol and Milli-Q water in order to remove excess thiol molecules.

\subsection{Formation of DPPC/Chol (70:30 mol\%) SLB on gold previously modified with MUA SAM}

\subsubsection{Preparation of vesicles}

Lipids, initially in chloroform, were dried under a nitrogen flow and then overnight vacuum. Subsequently, they were hydrated with Hepes buffer to a final concentration of $1 \mathrm{mM}$. The large unilamellar vesicles (LUV) suspension was prepared as previously reported [29], by the extrusion method using polycarbonate filters with $400 \mathrm{~nm}$ diameter pores (Nuclepore, Whatman).

\subsubsection{Vesicles deposition}

For the formation of the SLB, $150 \mu \mathrm{L}$ of LUV suspension were deposited on the MUA-modified gold, prepared as described above, incubated for 1 hour at $60^{\circ} \mathrm{C}$ and left to cool down at room temperature $\left(23^{\circ} \mathrm{C}\right)$ for $1 \mathrm{~h}$. Then they were washed several times at room temperature with Hepes buffer.

\subsection{Preparation of DPPC/cholesterol/decanethiol (65:30:5 mol\%) SLB on bare gold}

A stock solution of decanethiol in ethanol was also used and the required amount was mixed with the lipid solutions and dried together. The subsequent procedure to prepare and deposit LUV was identical to the one described above, except that this time bare gold instead of MUA-modified substrate was employed.

\subsection{Preparation of the activated surface}

After the lipid bilayer (DPPC/cholesterol/decanethiol) had been assembled, the substrate was submerged into a $0.2 \mathrm{M}$ EDC and $0.05 \mathrm{M}$ NHS solution for one hour and then rinsed with Hepes buffer.

\subsection{Ellipsometry}

Ellipsometric measurements were performed in air at an incidence angle of $70^{\circ}$ using a SE 400 Ellipsometer (Sentech Instruments) equipped with a He-Ne laser $(632.8 \mathrm{~nm})$. The thickness of the lipid film was estimated as reported in the literature $[5,27]$ fixing the real part of the refractive index at $1.5[29,30]$. The very low 
extinction coefficient values $(k)$ obtained in this work ranged from 0.03 to 0.11 , which indicates nearly transparent films, as expected for thin organic layers [27]. A thorough rinse with buffer and water (and ethanol in the case of MUA SAM deposition) and ellipsometric measurements were done between each modification step. SLB modified surfaces were incubated with $0.1 \mathrm{mg} / \mathrm{mL} \operatorname{IgG}$ in PBS for $1 \mathrm{~h}$ at room temperature. Finally, the gold substrates were immersed in $0.1 \mathrm{mg} / \mathrm{mL}$ anti-IgG in PBS for another hour.

\subsection{Surface plasmon resonance}

SPR measurements were carried out in a BIOSUPLAR $400 \mathrm{~T}$ compact SPR sensor manufactured by "Analytical $\mu$-Systems" coupled to a peristaltic pump from ISMATEC at a flow of $50 \mu \mathrm{L} / \mathrm{min}$.

After phosphate group activation, the gold slide was mounted onto the SPR equipment and $0.1 \mathrm{mg} / \mathrm{mL} \operatorname{IgG}$ was pumped into the system and left to incubate for, approximately, one hour. After PBS washing, $0.1 \mathrm{mg} / \mathrm{mL}$ anti-IgG was delivered into the cell. SPR experiments were conducted after a stable baseline was acquired. Data shown are the representative behavior of at least 3 independent experiments and are presented as absolute differences in resonance units (RUs). The signal noise level was determined to be $\leq 1 \mathrm{RU}$.

\subsection{Atomic force microscopy}

AFM measurements were performed in air at $23 \pm 1{ }^{\circ} \mathrm{C}$ using a Multimode Nanoscope IIIa microscope (Digital Instruments, Veeco) in tapping mode at a scan rate close to $1.7 \mathrm{~Hz}$. For this purpose, etched silicon probes (TESP, with a resonance frequency close to $300 \mathrm{kHz}$, Bruker) were used. All images were obtained after thoroughly rinsing with buffer solution and water and drying under $\mathrm{N}_{2}$ flow. Au(111) substrates modified with DPPC/Chol/decanethiol (65:30:5 mol\%) SLB were imaged after incubation with $0.1 \mathrm{mg} / \mathrm{mL}$ IgG (one hour) and $0.1 \mathrm{mg} / \mathrm{mL}$ anti-IgG (one hour). A set of, at least, three independent experiments was performed for each system.

\section{Results and discussion}

\subsection{Performance of SLB (DPPC/Chol. 70:30 mol\%) deposited onto a MUA SAM as a sensing interface}

A lipid bilayer was deposited on gold surfaces modified with MUA, the SAM typically used for these purposes [5,29,31], and its ability to prevent the nonspecific adsorption of BSA and IgG was tested. MUA SAM alone, as expected, did not prevent nonspecific adsorption of IgG (Fig. 2a), and in these circumstances a protein film with an estimated thickness $(d)$, by ellipsometry, of $\sim 3.4 \mathrm{~nm}$ was formed (Table 1). Nonspecific adsorption of IgG occurs also on bare gold but in a lower extent $\sim 2.1 \mathrm{~nm}$ [32]. However, SPR results show that the SLB (DPPC/Chol, 70:30) prepared on MUA-modified gold clearly inhibited the nonspecific adsorption of proteins (IgG and BSA) (Fig. 2b). Also, by ellipsometry, a thickness of $\sim 5.2 \mathrm{~nm}$ was estimated for the SLB, which is in good agreement with the one expected [29]. Moreover, ellipsometry also shows that only a negligible amount of IgG adsorbed on the SLB (Table 1). These results demonstrate that the liquid ordered lipid bilayer is very efficient in preventing undesired physical adsorption without the need of using blocking buffer or other agents. It has been reported a partial ability of a lipid film to suppress nonspecific adsorption of proteins [33,34] in other substrates, namely poly(dimethylsiloxane) [35], poly(methyl methacrylate) [33], silicon [34] and even gold [36]. However, the extent of suppression of the liquid ordered bilayer used is virtually complete.

Another crucial feature of lipid-based platforms is their stability and some authors found that the lipid film is removed upon rinsing with PBS buffer [33]. The stability of the bilayer (DPPC/Chol.
(70:30) on MUA-modified gold) illustrated in Fig. 2c was also compromised when high protein concentration solutions were used, as observed for casein-based BB. A great decrease in SPR signal was detected after $B B$ is passed through the channel, which points to the removal of the SLB formed onto MUA-modified gold. The main forces mediating the attachment of the SLB to the MUA-modified gold are electrostatic interactions, which may be disrupted upon $\mathrm{BB}$ solution is added.

\subsection{Immunosensing interface on decanethiol-containing lipid membrane}

To increase the stability of the SLB, $5 \mathrm{~mol} \%$ of decanethiol (DPPC/Chol/decanethiol 65:30:5 mol\%) was incorporated in the liposomes that were deposited on the substrate, allowing to form directly on bare gold a thiol-anchored SLB (Fig. 3). In this situation, the decanethiol molecules diluted in the membrane can establish a strong $\mathrm{Au}-\mathrm{S}$ bond to the gold surface in addition to the van der Waals interactions with adjacent lipid molecules, promoting a stable adhesion of the SLB to the metal surface. In Fig. 3a it is shown that a pure decanethiol SAM per se does not prevent nonspecific adsorption of $\operatorname{IgG}$, as proved by ellipsometry $(d \sim 3.6 \mathrm{~nm}$, Table 1$)$. In the supported lipid bilayer assembly decanethiol molecules should be present mostly in the bottom leaflet due to the high affinity of thiol groups for gold, thus most of the decanethiol present in the top leaflet in the vesicles should be transferred to the bottom one. This assumption is supported by the effective protection of this three-component bilayer interface against undesired nonspecific interactions with proteins (IgG and BB) (Fig. 3b). Even when BB is added, the change in the SPR signal was insignificant, after washing with PBS. Again, ellipsometry corroborates SPR data since only an irrelevant change in thickness was detected (Table 1). This reinforces the ability of the SLB to prevent nonspecific interactions, and also its strong stability against both a continuous flow and highly concentrated protein solutions.

With this method of lipid deposition, a continuous, planar and compact lipid bilayer on a gold substrate was readily formed. In fact, the lipid film thickness estimated was $5.5 \mathrm{~nm}$ (Table 1), in excellent agreement with the values previously obtained by us for gold-supported lipid bilayers [5,29]. It also matches the data reported for a liquid ordered phase of DPPC/Chol [37,38]. Moreover, the inhibition of protein nonspecific adsorption points to a fully covered gold surface, without the need of previous surface modification or of thiolipids. Concurrently, AFM images showed the formation of planar and defect-free lipid films on the gold surfaces used for both SPR (Fig. 4a,b) and ellipsometric (Fig. 4c,d) experiments and the absence of unfused vesicles. The gold substrates used in these experiments display distinct morphological properties. The thin layer gold $(200 \mathrm{~nm})$ with a predominant $\mathrm{Au}(111)$ crystallographic orientation, used for AFM and ellipsometry, has already proved to be suitable for SLB preparation [9,29], and is characterized by the presence of flat areas with monoatomic terraces, whereas $50 \mathrm{~nm}$ thick SPR gold is comprised of small $(15-20 \mathrm{~nm})$ globular grains [4]. Despite the different morphologies of the two gold substrates, which in the case of SPR type of substrates has been reported to hinder the formation of SLB from lipid vesicles, uniform, continuous and defect-free SLB were formed on both gold surfaces (Fig. 4). On the rougher SPR gold it is clear that the grain boundaries of gold become less evident proving the deposition of the lipid layer. The absence of unfused vesicles in any of the surfaces, together with the estimated thickness and SPR performance, as inhibiting protein non-specific adsorption, are consistent with the formation of a uniform and planar lipid bilayer.

The three-component lipid platform was then evaluated regarding its ability as an immunosensing interface. To this end, the phosphate groups of the phospholipids were activated taking 
Au-MUA

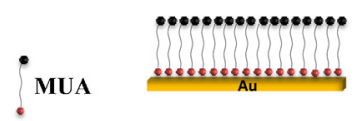

a)

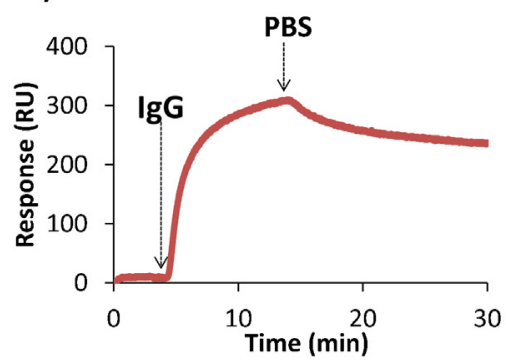

Au-MUA/ SLB (DPPC/CHOL 70:30)

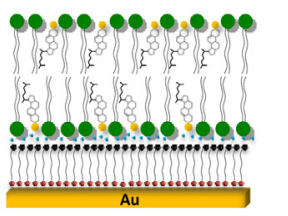

b)

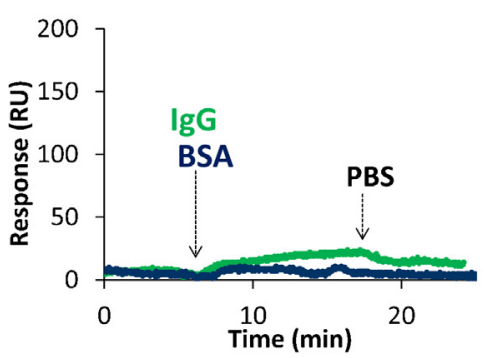

c)

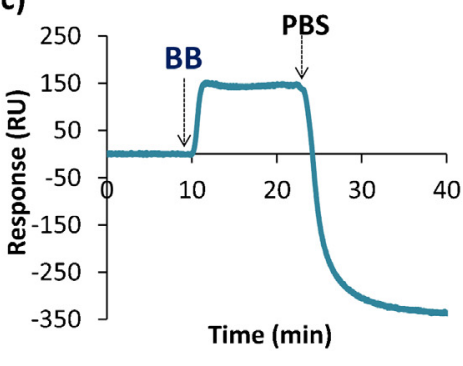

Fig. 2. Real-time SPR curves of the interaction of (a) IgG with Au/MUA; (b) IgG or BSA and (c) BB with DPPC/Chol (70:30) SLB deposited on Au/MUA.

Table 1

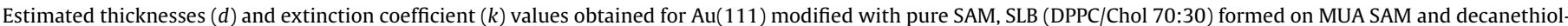

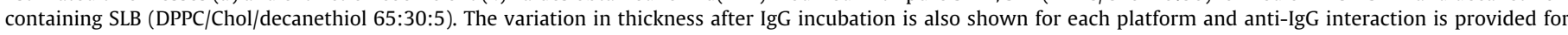
activated SLB (DPPC/Chol/decanethiol 65:30:5).

\begin{tabular}{|c|c|c|c|c|c|c|}
\hline \multirow[t]{3}{*}{ Surface } & \multicolumn{6}{|c|}{ Ellipsometry } \\
\hline & \multicolumn{2}{|l|}{ SLB } & \multicolumn{2}{|l|}{ IgG } & \multicolumn{2}{|l|}{ Anti-IgG } \\
\hline & $d(\mathrm{~nm})$ & $k$ & $\Delta d(\mathrm{~nm})$ & $k$ & $\Delta d(\mathrm{~nm})$ & $k$ \\
\hline Au-MUA & $0.8 \pm 0.1$ & $0.05 \pm 0.02$ & $3.4 \pm 0.5$ & $0.09 \pm 0.02$ & - & - \\
\hline Au-decanethiol & $0.6 \pm 0.1$ & $0.06 \pm 0.04$ & $3.6 \pm 0.7$ & $0.11 \pm 0.02$ & - & - \\
\hline Au-MUA/SLB (DPPC/Chol) & $5.2 \pm 0.9$ & $0.04 \pm 0.02$ & $0.2 \pm 0.1$ & $0.03 \pm 0.02$ & - & - \\
\hline Au-DPPC/Chol/decanethiol No activation & $5.5 \pm 0.6$ & $0.03 \pm 0.03$ & $0.1 \pm 0.1$ & $0.03 \pm 0.02$ & - & - \\
\hline Au-DPPC/Chol/decanethiol Activation & $5.5 \pm 0.6$ & $0.03 \pm 0.03$ & $3.5 \pm 0.6$ & $0.03 \pm 0.01$ & $7.0 \pm 0.6$ & $0.03 \pm 0.01$ \\
\hline
\end{tabular}

a) Au-DECANETHIOL

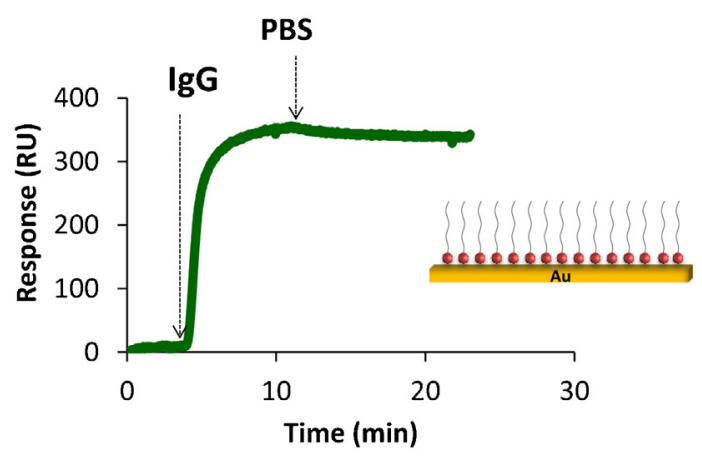

b) Au-SLB (DPPC/CHOL/DECANETHIOL 65:30:5)

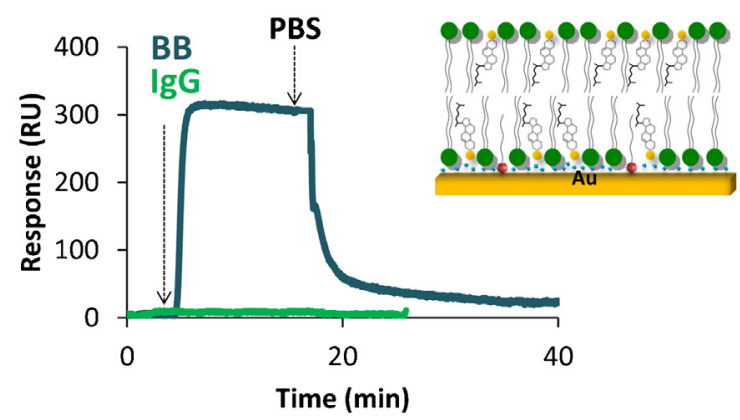

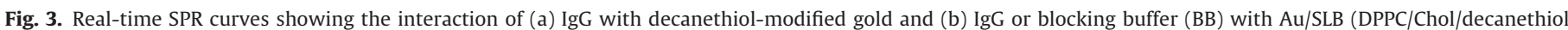
65:30:5).

advantage of EDC and NHS chemistry, a similar approach to that already employed by Zhao and co-workers [26] to covalently attach double-stranded DNA to amine terminated SAMs. To the best of our knowledge this is the first time that this coupling agents are used to activate phosphate groups in lipids for immunosensing. After this reaction, modified phosphate groups become reactive toward amine groups, enabling the covalent coupling between lipid head and antibodies. The modified gold substrate was then mounted in the SPR cell and a solution containing the antibody IgG was delivered into the system. In contrast with the observations in the absence of the activation step (Fig. 3b), after the EDC/NHS activa- tion, a strong and stable change in SPR signal occurred $(\Delta R U=186$, Fig. 5a; versus $\Delta R U \sim 0$, Fig. $3 \mathrm{~b}$ ) evolving to a plateau (Fig. 5a). The SPR signal remained unaltered after the usual washing step with PBS buffer, showing that IgG is stably linked to the lipid surface. Subsequent pumping of $0.1 \mathrm{mg} / \mathrm{mL}$ of anti-IgG into the system generated a great response in SPR signal $(\Delta R U=390)$ due to antibody/antigen biorecognition. Importantly, no significant changes in the SPR signal occur after PBS with $0.05 \%$ of Tween 20 is flowed into the system containing only the SLB interface or anti-IgG/IgG coupled to the SLB (inset in Fig. 5a). This shows not only that the SLB assembly is very stable regarding the use of a low percentage of 

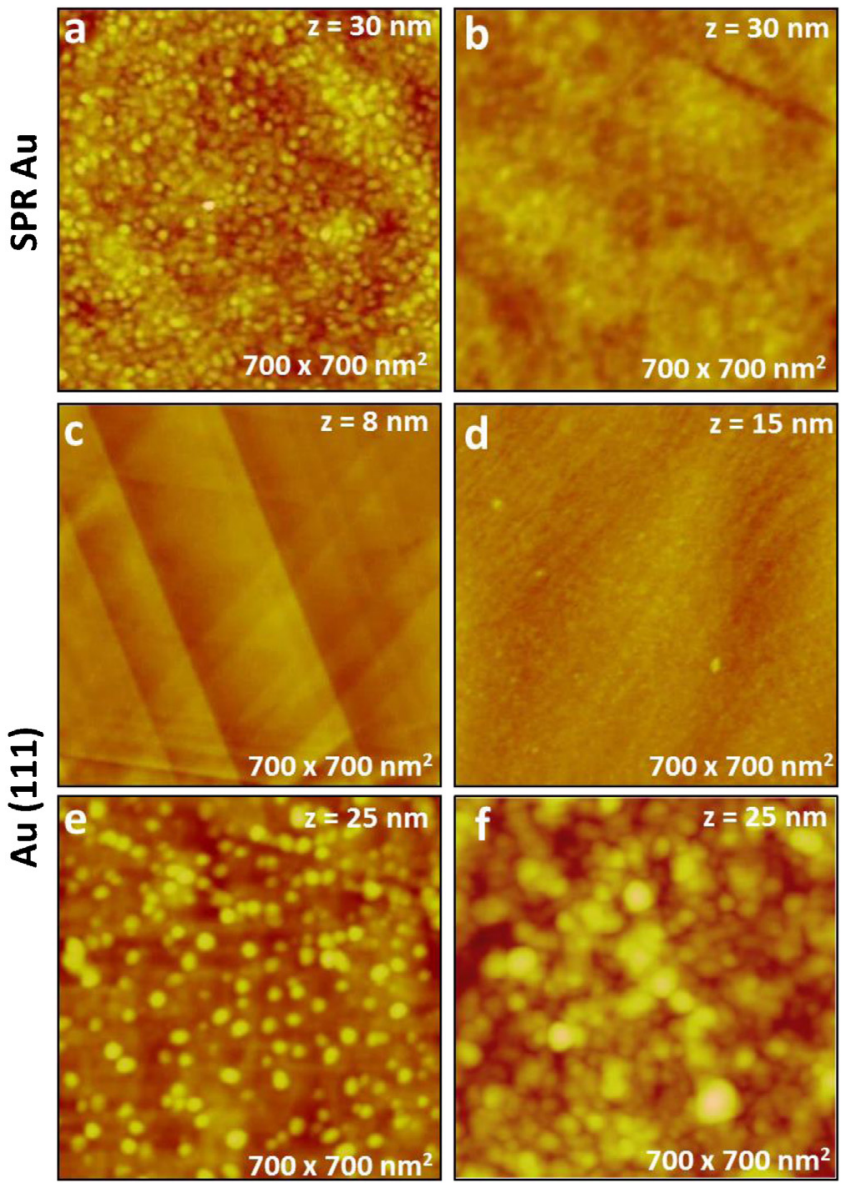

Fig. 4. AFM topographic images of (a) bare and (b) modified gold with SLB (DPPC/Chol/decanethiol 65:30:5) used in SPR experiments. AFM images of (c) bare and modified $\mathrm{Au}$ (111) with (d) SLB, (e) SLB/IgG and (f) SLB/IgG/anti-IgG. surfactant but also that solely specific interactions are established upon anti-IgG/IgG biorecognition. These results strengthened the advantage of using liquid ordered bilayers, in particular DPPC/Chol, which is very stable and much more resistant to detergent solubilization than most fluid lipid bilayers.

The ellipsometric measurements (Fig. 5b,c and Table 1) performed on modified $\mathrm{Au}(111)$ with DPPC/Chol/decanethiol (65:30:5 mol\%) further demonstrated the inhibition of nonspecific adsorption, since there was only a negligible variation of the total thickness ( $\Delta d \sim 0.1 \mathrm{~nm}$ ) after incubation with $\operatorname{IgG}$. However, upon EDC/NHS activation of phosphate groups of SLB, a change in thickness of $\sim 3.5 \mathrm{~nm}$ was obtained after IgG incubation. Further interaction with anti-IgG promoted a significant variation in ellipsometric parameters corresponding to $\Delta d \sim 7 \mathrm{~nm}$, which expresses the recognition of ca. two anti-IgG molecules per IgG, confirming the suitability of this platform to monitor antibody-antigen reactions. In the immunosensing interface we used anti-IgG $(\mathrm{H}+\mathrm{L})$ secondary antibodies, which react with both heavy $(\mathrm{H} ; \mathrm{Fc})$ and light $\left(\mathrm{L} ; \mathrm{F}\left(\mathrm{ab}^{\prime}\right) 2 / \mathrm{Fab}\right)$ portions of the immobilized primary IgG, thus up to two anti-IgG molecules were expected to bind per immobilized IgG. The high ratio of anti-IgG/IgG detected by both SPR and ellipsometry confirms such effective, bivalent, binding and also suggests that the simple methodology employed may favor a preferential orientation of immobilized antibodies. Moreover, the effective multivalent biding also indicates sufficient spacing between IgG and no steric hindrance by the constructed layer.

AFM images of modified gold surfaces were also acquired upon IgG coupling (Fig. 4e) and after anti-IgG specific binding (Fig. 4f). They show the presence of globular structures with a diameter $\sim 20 \mathrm{~nm}$ after incubation with IgG and larger aggregates upon antiIgG interaction. Thus, AFM data corroborates the high anti-IgG/IgG ratio already detected by both SPR and ellipsometric measurements.

To the best of our knowledge this is the first report that employs multicomponent vesicles of a common phospholipid, cholesterol and decanethiol to form a planar and uniform bilayer on gold.

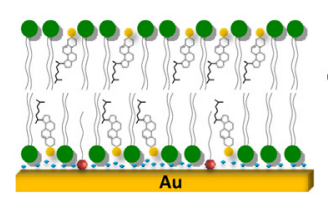

a)

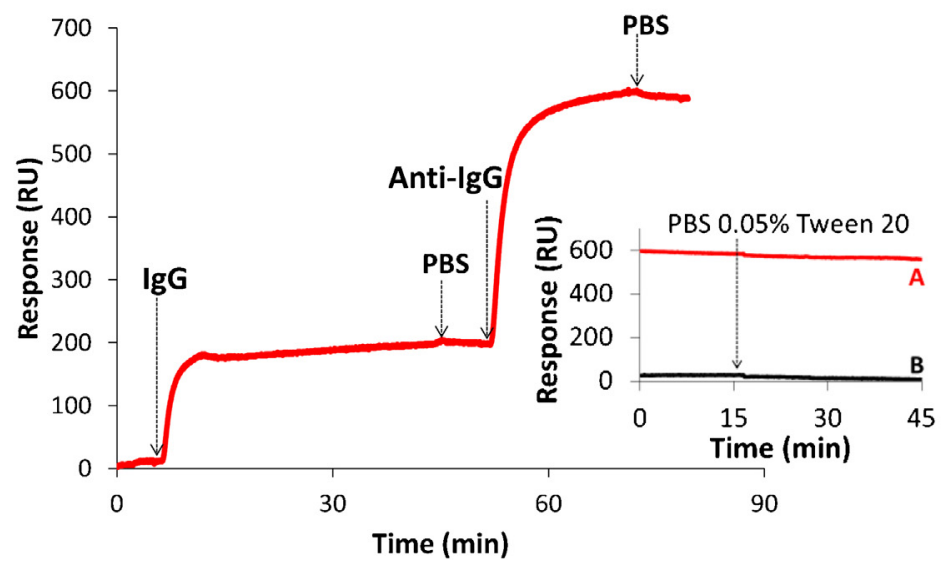

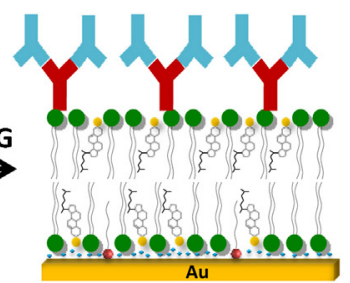

b)

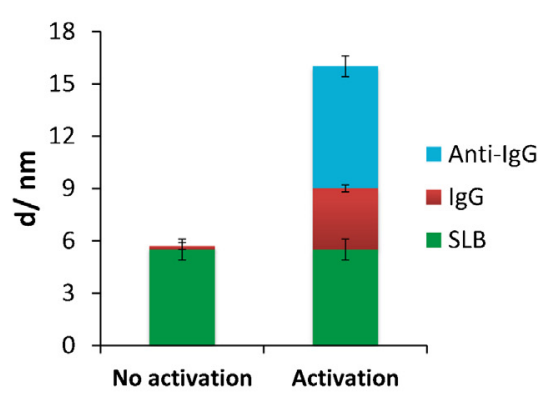

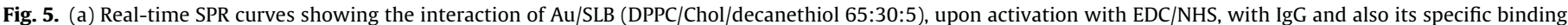

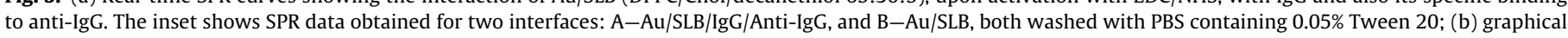
representation of the thickness estimated by ellipsometry for each modification step (mean \pm standard deviation). 
Previously to this work, there was only one attempt to deposit alkanethiol containing liposomes on gold [39]. However in that study, the authors used a binary system composed of a glycolipid and higher thiol concentration ( $30 \mathrm{~mol} \%$ ), which lead to vesicle adsorption and flattening of the vesicles without fusion and concomitant formation of SLB, as shown by AFM imaging.

\section{Conclusions}

With this report we present a lipid-based biointerface which combines a number of important characteristics when developing a biosensor: (i) biomimetic environment, (ii) use of common and commercially available reagents, (iii) simple preparation, (iv) ability to block nonspecific interaction with serum proteins, (v) robust arrangement regarding a continuous flow of buffer solutions (even in the presence of surfactant) or highly concentrated protein solutions, very promising when microfluidic devices are envisaged, and finally, (vi) straightforward activation of lipid bilayers for the covalent coupling of the recognition antibody.

As far as we are concerned this method has not been employed previously for the covalent attachment between phospholipids and proteins. Its simplicity, due to the fact that all SLB components are mixed together and only the usual steps for the preparation of SLB need to be carried out, without the requirement of previous surface modification or the addition of extra components such as blocking agents, makes this an attractive alternative to other lipid-based biosensing platforms. In addition, the direct linkage of antibodies to the lipid head groups further simplify the method, as there is no need of an extra component within the bilayer (ex. with appended $\mathrm{COOH}$ groups), which can affect the natural lipid organization and compactness.

\section{Conflict of interest}

The authors declare no competing financial interest.

\section{Acknowledgments}

This work was financed by the $7^{\text {th }}$ Sino-Portugal Scientific and Technological Cooperation of 2013-2015 and by Portuguese national funds through FCT: Ph.D. fellowships: SFRH/BD/64442/2009, SFRH/BD/70673/2010 grants PEst OE/QUI/UI0612/2013 and PEst 2015-2020 (UID/Multi/00612/2013), and IF 2012/2013 initiatives (POPH, Fundo Social Europeu).

\section{References}

[1] E. Reimhult, K. Kumar, Membrane biosensor platforms using nano- and microporous supports, Trends Biotechnol. 26 (2008) 82-89.

[2] W. Ma, Y.L. Ying, L.X. Qin, Z. Gu, H. Zhou, D.W. Li, T.C. Sutherland, H.Y. Chen, Y.T. Long, Investigating electron-transfer processes using a biomimetic hybrid bilayer membrane system, Nat. Protoc. 8 (2013) 439-450.

[3] M.B. Fritzen-Garcia, V.C. Zoldan, I.R.W.Z. Oliveira, V. Soldi, A.A. Pasa, T.B. Creczynski-Pasa, Peroxidase immobilized on phospholipid bilayers supported on au(111) by DTT self-assembled monolayers: application to dopamine determination, Biotechnol. Bioeng. 110 (2013) 374-382.

[4] J.T. Marques, R.F.M. de Almeida, A.S. Viana, Lipid bilayers supported on bare and modified gold-formation, characterization and relevance of lipid rafts, Electrochim. Acta 126 (2014) 139-150.

[5] J.T. Marquês, A.S. Viana, R.F.M. de Almeida, A biomimetic platform to study the interactions of bioelectroactive molecules with lipid nanodomains, Langmuir 30 (2014) 12627-12637.

[6] A. Coutable, C. Thibault, J. Chalmeau, J.M. Francois, C. Vieu, V. Noireaux, E. Trevisiol, Preparation of tethered-lipid bilayers on gold surfaces for the incorporation of integral membrane proteins synthesized by cell-free expression, Langmuir 30 (2014) 3132-3141.

[7] J. Lipkowski, Chapter one-biomimetic membrane supported at a metal electrode surface: a molecular view, in: I. Aleš, V.K. Chandrashekhar (Eds.), Advances in Planar Lipid Bilayers and Liposomes, Academic Press, 2014, pp. $1-49$.
[8] E. Gizeli, M. Liley, C.R. Lowe, H. Vogel, Antibody binding to a functionalized supported lipid layer: a direct acoustic immunosensor, Anal. Chem. 69 (1997) 4808-4813.

[9] J. Lipkowski, Building biomimetic membrane at a gold electrode surface, Phys. Chem. Chem. Phys. 12 (2010) 13874-13887.

[10] J.A. Jackman, W. Knoll, N.J. Cho, Biotechnology applications of tethered lipid bilayer membranes, Materials 5 (2012) 2637-2657.

[11] S. Kissler, S. Pierrat, T. Zimmermann, H. Vogt, H.K. Trieu, I. Koper, CMOS based capacitive biosensor with integrated tethered bilayer lipid membrane for real-time measurements, Biomed. Eng. 57 (2012)

[12] I. Pfeiffer, B. Seantier, S. Petronis, D. Sutherland, B. Kasemo, M. Zach, Influence of nanotopography on phospholipid bilayer formation on silicon dioxide, J. Phys. Chem. B 112 (2008) 5175-5181.

[13] F. Albertorio, A.J. Diaz, T.L. Yang, V.A. Chapa, S. Kataoka, E.T. Castellana, P.S Cremer, Fluid and air-stable lipopolymer membranes for biosensor applications, Langmuir 21 (2005) 7476-7482

[14] A. Lundquist, S.B. Hansen, H. Nordstrom, U.H. Danielson, K. Edwards, Biotinylated lipid bilayer disks as model membranes for biosensor analyses, Anal. Biochem. 405 (2010) 153-159.

[15] I. Koper, Insulating tethered bilayer lipid membranes to study membrane proteins, Mol. Biosyst. 3 (2007) 651-657.

[16] T. Hianik, M. Snejdarkova, L. Sokolikova, E. Meszar, R. Krivanek, V. Tvarozek, I. Novotny, J. Wang, Immunosensors based on supported lipid membranes, protein films and liposomes modified by antibodies, Sens. Actuators B 57 (1999) 201-212.

[17] Y. Ishizuka-Katsura, T. Wazawa, T. Ban, K. Morigaki, S. Aoyama, Biotin-containing phospholipid vesicle layer formed on self-assembled monolayer of a saccharide-terminated alkyl disulfide for surface plasmon resonance biosensing, J. Biosci. Bioeng. 105 (2008) 527-535.

[18] M. Brzozowska, B.P. Oberts, G.J. Blanchard, J. Majewski, P. Krysinski, Design and characterization of novel tether layer for coupling of a bilayer lipid membrane to the surface of gold, Langmuir 25 (2009) 9337-9345.

[19] J.K.R. Kendall, B.R.G. Johnson, P.H. Symonds, G. Imperato, R.J. Bushby, J.D. Gwyer, C. van Berkel, S.D. Evans, L.J.C. Jeuken, Effect of the structure of cholesterol-based tethered bilayer lipid membranes on ionophore activity, Chemphyschem 11 (2010) 2191-2198.

[20] J.H. Ipsen, G. Karlstrom, O.G. Mouritsen, H. Wennerstrom, M.J. Zuckermann, Phase-equilibria in the phosphatidylcholine-cholesterol system, Biochim. Biophys. Acta 905 (1987) 162-172.

[21] R.F.M. De Almeida, J. Borst, A. Fedorov, M. Prieto, A.J.W.G. Visser, Complexity of lipid domains and rafts in giant unilamellar vesicles revealed by combining imaging and microscopic and macroscopic time-resolved fluorescence, Biophys. J. 93 (2007) 539-553.

[22] M.R. Vist, J.H. Davis, Phase-equilibria of cholesterol dipalmitoylphosphatidylcholine mixtures-h-2 nuclear magnetic-resonance and differential scanning calorimetry, Biochemistry 29 (1990) 451-464.

[23] D. Marsh, Liquid-ordered phases induced by cholesterol: a compendium of binary phase diagrams, Biochim. Biophys. Acta 1798 (2010) 688-699.

[24] M.J. Uline, I. Szleifer, Mode specific elastic constants for the gel, liquid-ordered, and liquid-disordered phases of DPPC/DOPC/cholesterol model lipid bilayers, Faraday Discuss. 161 (2013) 177-191.

[25] R.F.M. de Almeida, E. Joly, Crystallization around solid-like nanosized docks can explain the specificity, diversity, and stability of membrane microdomains, Front. Plant Sci. 5 (14) (2014).

[26] Y.D. Zhao, D.W. Pang, S. Hu, Z.L. Wang, J.K. Cheng, H.P. Dai, DNA-modified electrodes; part 4: optimization of covalent immobilization of DNA on self-assembled monolayers, Talanta 49 (1999) 751-756.

[27] Y. Niu, A.I. Matos, L.M. Abrantes, A.S. Viana, G. Jin, Antibody oriented immobilization on gold using the reaction between carbon disulfide and amine groups and its application in immunosensing, Langmuir 28 (2012) $17718-17725$.

[28] I. Almeida, A.C. Cascalheira, A.S. Viana, One step gold (bio) functionalisation based on CS2-amine reaction, Electrochim. Acta 55 (2010) 8686-8695.

[29] J.T. Marques, R.F.M. De Almeida, A.S. Viana, Biomimetic membrane rafts stably supported on unmodified gold, Soft Matter 8 (2012) 2007-2016.

[30] R.F.M. de Almeida, L.M.S. Loura, A. Fedorov, M. Prieto, Nonequilibrium phenomena in the phase separation of a two-component lipid bilayer Biophys. J. 82 (2002) 823-834

[31] S. Ip, J.K. Li, G.C. Walker, Phase segregation of untethered zwitterionic model lipid bilayers observed on mercaptoundecanoic-acid-modified gold by AFM imaging, Langmuir 26 (2010) 11060-11070.

[32] Z.H. Wang, A.S. Viana, G. Jin, L.M. Abrantes, Immunosensor interface based on physical and chemical immunogylobulin $\mathrm{G}$ adsorption onto mixed self-assembled monolayers, Bioelectrochemistry 69 (2006) 180-186.

[33] N.C.H. Le, V. Gubala, R.P. Gandhiraman, S. Daniels, D.E. Williams, Evaluation of different nonspecific binding blocking agents deposited inside poly(methyl methacrylate) microfluidic flow-cells, Langmuir 27 (2011) 9043-9051.

[34] K. Glasmästar, C. Larsson, F. Höök, B. Kasemo, Protein adsorption on supported phospholipid bilayers, J. Colloid Interface Sci. 246 (2002) 40-47.

[35] K.S. Phillips, Q. Cheng, Microfluidic immunoassay for bacterial toxins with supported phospholipid bilayer membranes on poly(dimethylsiloxane), Anal. Chem. 77 (2004) 327-334.

[36] A.L. Plant, M. Brighamburke, E.C. Petrella, D.J. Oshannessy, Phospholipid/alkanethiol bilayers for cell-surface receptor studies by surface plasmon resonance, Anal. Biochem. 226 (1995) 342-348. 
[37] L. Chen, Z. Yu, P.J. Quinn, The partition of cholesterol between ordered and fluid bilayers of phosphatidylcholine: a synchrotron X-ray diffraction study, Biochim. Biophys. Acta 1768 (2007) 2873-2881.

[38] J.T. Marques, A.S. Viana, R.F.M. De Almeida, Ethanol effects on binary and ternary supported lipid bilayers with gel/fluid domains and lipid rafts, Biochim. Biophys. Acta 1808 (2011) 405-414.
[39] C. Guo, P. Boullanger, L. Jiang, T. Liu, One-step immobilization of alkanethiol/glycolipid vesicles onto gold electrode: amperometric detection of concanavalin A, Colloids Surf. B 62 (2008) 146-150. 